\title{
Intrinsic Sizes of the W3 (OH) Masers via Short Time Scale Variability
}

\author{
Tanmoy Laskar ${ }^{1}$, W. M. Goss ${ }^{2}$ and B. Ashley Zauderer ${ }^{1}$ \\ ${ }^{1}$ Harvard University, 60 Garden St., Cambridge, MA - 02138, USA \\ email: tlaskar@cfa.harvard.edu \\ ${ }^{2}$ National Radio Astronomy Observatory, PO Box 0, Socorro, NM - 87801, USA \\ textitail: mgoss@aoc.nrao.edu
}

\begin{abstract}
We present a study of short time-scale variability of $\mathrm{OH}$ masers within a contiguous 15-hour Very Long Baseline Array observation of the high-mass star-forming region, W3 (OH). With an angular resolution of $\sim 7$ mas and a velocity resolution of $53 \mathrm{~m} \mathrm{~s}^{-1}$, we isolate emission from masers in the field into individual Gaussian-shaped components, each a few milliarcseconds in size. We compute dynamic spectra for individual maser features with a time resolution of 1 minute by fitting for the flux density of all sources in the field simultaneously in the $u \mathrm{v}$-domain. We isolate intrinsic maser variability from interstellar scintillation and instrumental effects. We find fluctuations in the maser line shape on time scales of 5 to 20 minutes, corresponding to maser column lengths of 0.5 to 2.0 Astronomical Units.
\end{abstract}

\section{Introduction}

Galactic $\mathrm{OH}$ masers have been found to be variable on a variety of time scales: from years (Caswell \& Vaile 1995) and months (e.g., Slysh et al. 2010) to days (Clegg \& Cordes 1991). The origin of this long-time scale variability is unknown, although proposed mechanisms have included changes in the physical conditions of the maser column (e.g., Zuckerman et al. 1972), sudden onset of pumping (Salem \& Middleton 1978), and radiative instabilities in the maser column (Scappaticci \& Watson 1992). Maser variability studies on time scales on the order of minutes are useful in probing the length of the maser column. Such studies have proved challenging, in part due to the effects of interstellar diffractive scintillation and the need to study spectrally-isolated masers.

Recently, Ramachandran et al. 2006 (hereafter R06) studied variability in the galactic star-forming region, W3 $(\mathrm{OH})$ on short $(\sim$ minute) timescales with archival VLBA data (Wright et al. 2004). They employed a statistical technique to separate the effects of interstellar diffractive scintillation, based on the expectation for variability arising from scintillation to have a wider correlation bandwidth compared to the maser line width. After accounting for scintillation, they found significant residual variability (modulation indices up to $100 \%$ ) on time scales of about 15-20 minutes in the line wings of three strong, spectrally-isolated masers at 1612, 1665 and $1720 \mathrm{MHz}$. Although this provided tantalizing evidence for intrinsic variability of $\mathrm{OH}$ masers, certain aspects of the data used made it unfavorable for a final and conclusive analysis: the data had non-uniformly spaced gaps whenever a calibrator was observed, making standard Fourier analysis on the time series difficult. Additionally, the velocity resolution of $90 \mathrm{~m} \mathrm{~s}^{-1}$ resulted in spectral blending, making it hard to find isolated maser sources for variability analysis.

We present the results of a variability study of $\mathrm{OH}$ masers in $\mathrm{W} 3(\mathrm{OH})$ using Very Long Baseline Array (VLBA) data obtained with a factor of 2 better velocity resolution compared to the data used by R06. We fit for all maser sources present in the field 

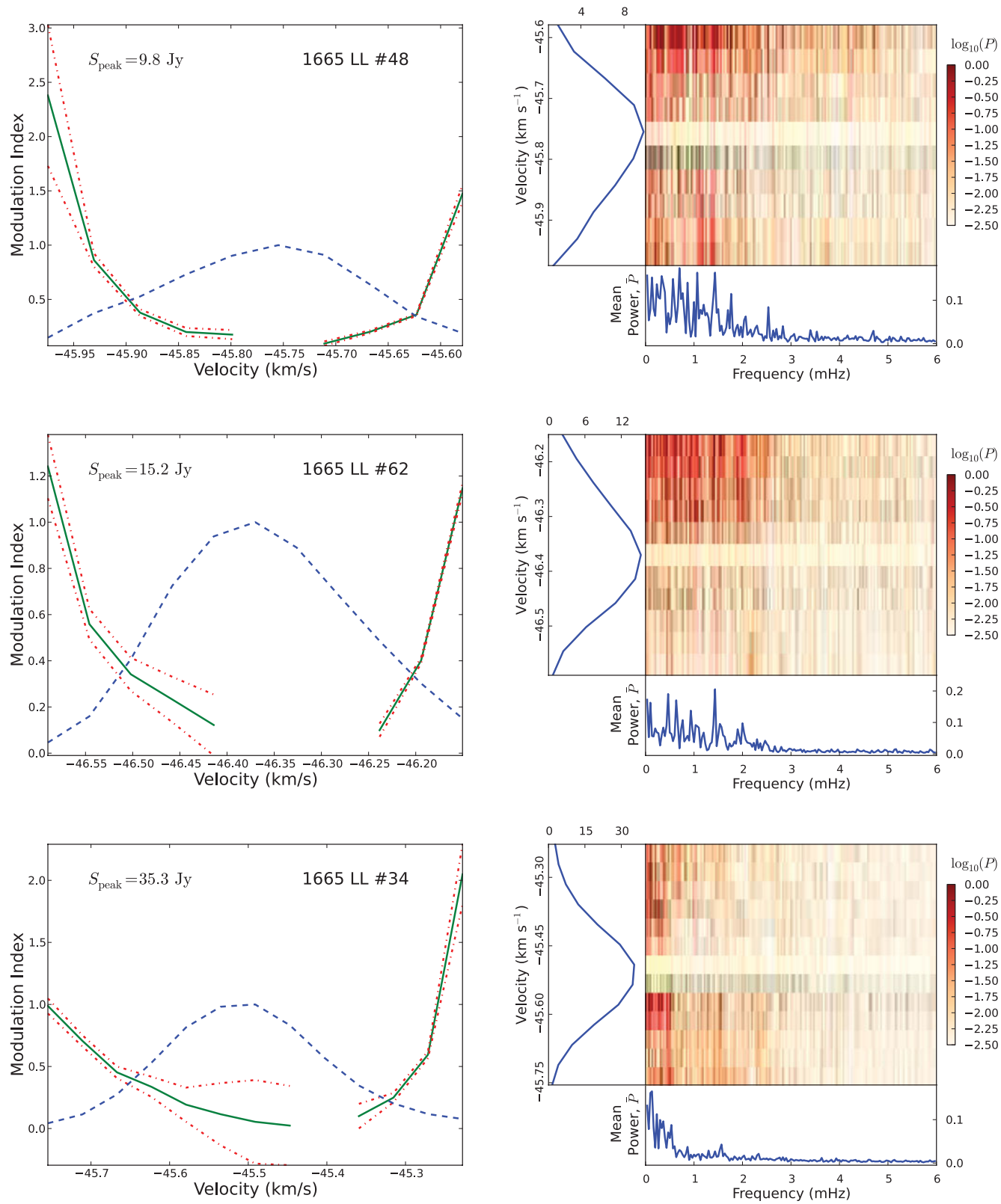

Figure 1. Left: Modulation index (solid green lines) and $\pm 1 \sigma$ uncertainties (dotted red lines), together with normalized spectral line profiles (blue dashed lines) for eight maser sources in W3 $(\mathrm{OH})$. The details of the masers used are presented in Table 1. All sources exhibit statistically significant variability in the line wings (modulation indices $\gtrsim 50 \%$ ). The line core appears not to be variable in all cases, confirming the results of Ramachandran et al. 2006. Right: Fluctuation power spectra for each source, obtained by Fourier transforming each channel in the dynamic spectrum and taking the square modulus. In each sub-figure, the $2 \mathrm{~d}$ frame is the velocity-resolved fluctuation spectrum in logarithmic units, the bottom panel is the velocity-averaged fluctuation spectrum in linear units, and the left panel is the average line profile in Jy/beam. The power spectra demonstrate a cut-off at high frequencies, corresponding to time scales of $5-20$ minutes. The $1665 \mathrm{MHz}$ Stokes LL sources (arranged here in order of increasing flux density) appear to show decreasing power at high fluctuation frequencies, indicating a possible increase in the time scale of variability with flux density. 

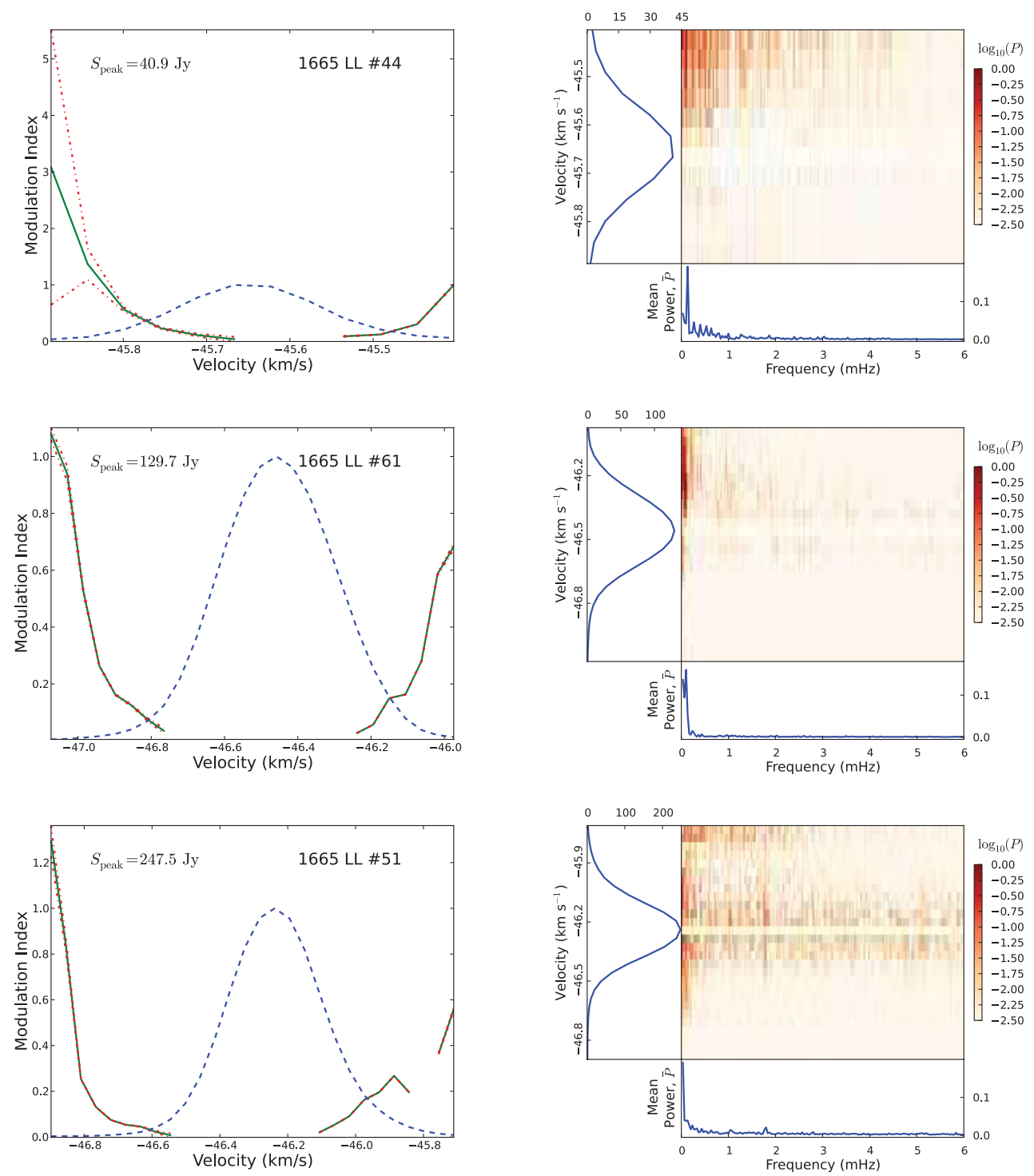

Figure 1. (Continued)

simultaneously directly in the $u \mathrm{v}$-domain, lifting the restriction of sources under study to be spectrally isolated, which greatly increases the number of masers available for this study. Using the statistical tools of R06, we find variability on time scales of $5 \sim$ 20 minutes with modulation indices between 50 and $100 \%$ for eight maser sources in $\mathrm{W} 3(\mathrm{OH})$, implying maser column lengths of 0.5 to 2 astronomical units.

\section{Observations and Data Analysis}

We obtained a 15-hour contiguous observation of W3 (OH) with the VLBA (all ten VLBA stations) on 2005 July 07 (VLBA program BR107) in all four ground state transitions with a velocity resolution of $\sim 53 \mathrm{~m} \mathrm{~s}^{-1}$. RFI was excised and the data were phase self-calibrated. In $4^{\prime \prime} \times 4^{\prime \prime}$ images (synthesized beam $6.7 \times 7.7 \mathrm{mas}$ ), we detected 

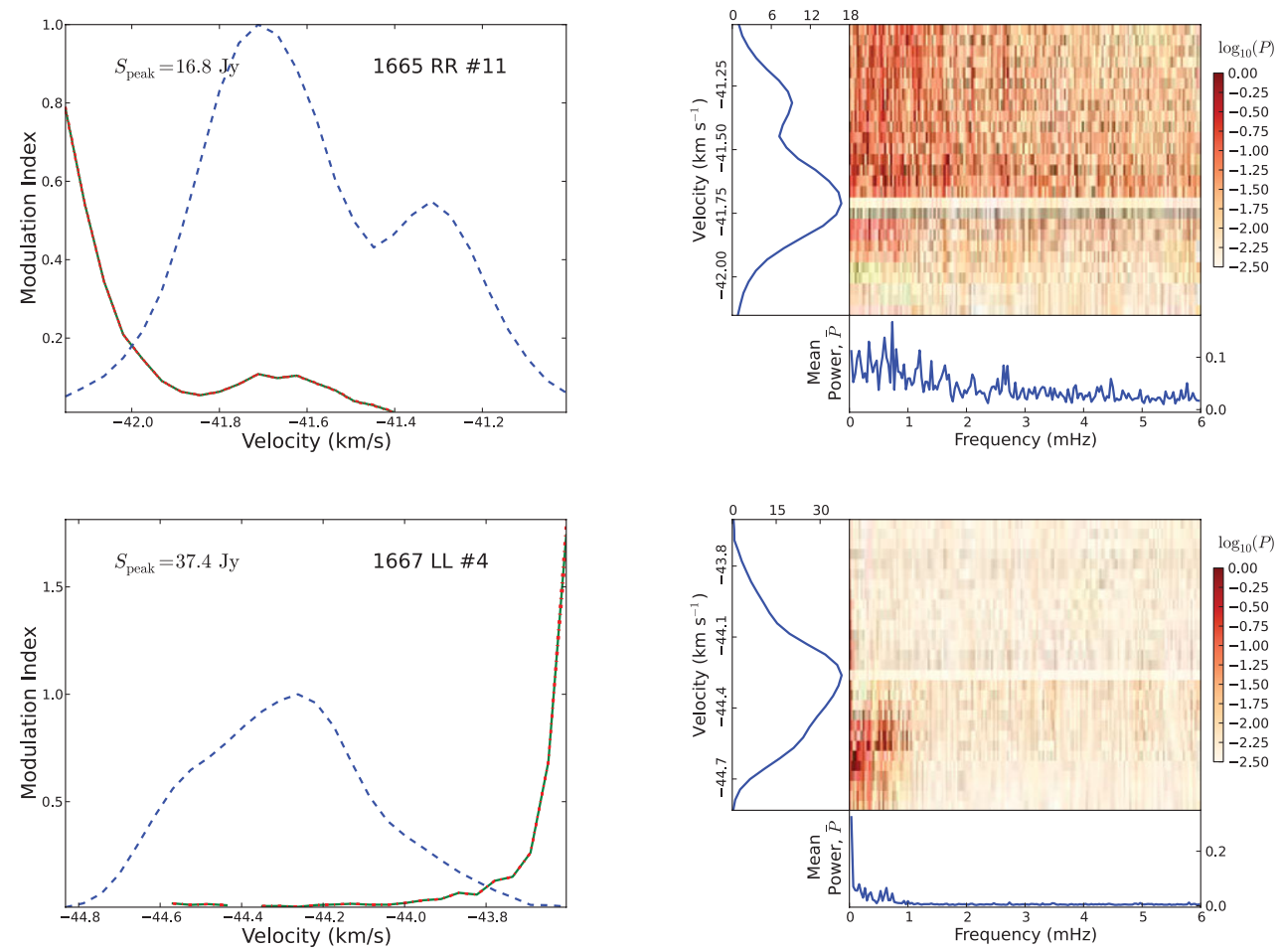

Figure 2. Similar to Figure 1, for a 1665 RR maser and a 1667 LL maser.

approximately 20 distinct maser sources at $1612 \mathrm{MHz}, 10$ at $1720 \mathrm{MHz}, 180$ at $1665 \mathrm{MHz}$ and 40 at $1667 \mathrm{MHz}$ brighter than $10 \sigma$. The noise level in our maps ranged from $\sim 30 \mathrm{mJy} /$ beam in line-free parts of the spectrum to $\sim 60 \mathrm{mJy} /$ beam in channels with strong masers.

Dynamic spectra for every source brighter than 1 Jy were generated by simultaneously fitting for the flux density in the $u \mathrm{v}$ domain in the entire 256-channel spectral cube at 1 minute intervals. The fitting was carried out in the $u \mathrm{v}$ domain since the sparse $u \mathrm{v}-$ coverage at short integrations results in very poor image fidelity with large side-lobe responses whenever multiple sources are present in a single spectral channel, as is the case for the majority of channels in the data cube. With this simultaneous fit for all maser sources, we are not restricted to analyzing only spectrally-isolated sources and are able to extend the work by R06 to all strong sources in the entire data set. For the 1-minute fits, we fixed the positions and shape properties of each maser component to the values determined by imaging the full 15-hour observation.

We used statistical techniques from R06 to determine modulation indices across the line profile for 8 maser sources (Figure 1, left panels). These modulation indices imply significant $(\sim 100 \%)$ variability in the line wings. In each case, the modulation index is consistent with zero near the peak of the line, indicating that the line core is not significantly variable. Both of these results are in agreement with the conclusions of R06.

\section{Fluctuation Analysis}

To estimate the time scale corresponding to narrow band variability, the contribution of broad band variability to the dynamic spectrum must be removed. We achieve this by dividing the observed dynamic spectrum by the flux density in the reference channel 
Table 1. List of masers used for the present analysis.

\begin{tabular}{cccccc}
\hline $\begin{array}{c}\text { Transition } \\
(\mathbf{M H z})\end{array}$ & Polarization & $\begin{array}{c}\text { Source } \\
\text { Number }^{1}\end{array}$ & $\begin{array}{c}\text { Velocity }^{2} \\
\left(\mathbf{k m ~ s}^{-1}\right)\end{array}$ & $\begin{array}{c}\text { RA offset }^{3} \\
\text { arcsec }^{3}\end{array}$ & $\begin{array}{c}\text { Dec offset }^{3} \\
\text { arcsec }^{3}\end{array}$ \\
\hline 1665 & LL & 34 & -45.51 & -0.595 & -1.918 \\
1665 & LL & 44 & -45.65 & -0.711 & -1.673 \\
1665 & LL & 48 & -45.77 & -0.851 & -0.618 \\
1665 & LL & 51 & -46.24 & -1.024 & -0.123 \\
1665 & LL & 61 & -46.45 & -0.960 & -0.103 \\
1665 & LL & 62 & -46.37 & -0.168 & -0.044 \\
1665 & RR & 11 & -41.63 & -0.141 & -1.185 \\
1667 & LL & 4 & -44.31 & -0.386 & -1.886 \\
\hline
\end{tabular}

Notes:

${ }^{1}$ The source numbers given here are internal to our analysis, and are likely to change in a future publication.

${ }^{2}$ LSR Velocity of the line centroid, determined by fitting a single Gaussian to the spectral line.

${ }^{3}$ Right Ascension and Declination offsets (J2000.0) from the phase center, corresponding to the location of the flux-weighted centroid of the maser line. Since our data were self-calibrated, the exact location of the phase center in RA and Dec is uncertain. Preliminary analysis suggests that our offset coordinate system is comparable (within about 10 mas) to that used by Fish et al. 2006.

defined by the peak of the average line profile. This is justified since the peak of the line does not exhibit statistically significant narrow band variations, as apparent from the modulation indices (Figure 1, left panels). We compute the fluctuation power spectrum for each channel by Fourier transforming the time series in each channel individually, followed by taking the square modulus. Our preliminary results are shown in Figure 1 (right panels). Excess power is evident at low frequencies, below about $3 \times 10^{-3} \mathrm{~Hz}$, corresponding to a minimum variability time scale of about 5 minutes. The power spectra for the $1665 \mathrm{MHz}$ Stokes LL masers also hint at a possible decrease in power at high fluctuation frequencies (corresponding to an increase in the variability time scale), with increasing flux density of the maser line.

In summary, we confirm the results of R06 and find fluctuations in the maser line shape on time scales of 5 to 20 minutes, corresponding to maser columns of 0.5 to $2.0 \mathrm{AU}$. In a future publication, we aim to extend our analysis to other maser sources in the field and to compare variability between masers in Zeeman pairs.

The National Radio Astronomy Observatory is a facility of the National Science Foundation operated under cooperative agreement by Associated Universities, Inc.

\section{References}

Baudry, A., Desmurs, J. F., Wilson, T. L., \& Cohen, R. J. 1997, A $\& A$, 325, 255

Caswell, J. L. \& Vaile R. A. 1995, MNRAS, 273, 328

Clegg, A. W. \& Corders J. M. 1991, ApJ, 374, 150

Fish, V. L., Brisken W. F., \& Sjouwerman L. O. 2006, ApJ, 647, 418

Ramachandran, R., Deshpande, A. A., \& Goss, W. M. 2006, ApJ, 653, 1314

Rickard, L. J., Palmer, P., \& Zuckerman, B. 1975, ApJ, 200, 6

Salem, M. \& Middleton, M. S. 1978, MNRAS, 183, 491

Scappaticci, G. A. \& Watson, W. D. 1992, ApJ, 400, 351

Slysh, V. I., Alakoz, A. V., \& Migenes, V. 2010, MNRAS, 404, 1121

Wright, M. M., Gray, M. D., \& Diamond, P. J. 2004, MNRAS, 350,1253

Zuckerman, B. Yen, J. L., Gottlieb, C. A., \& Palmer, P. 1972, ApJ, 177, 59 